Australia on the joint development of this resource, which lies geographically between the two countries.

The government has decided that Portuguese and Tetum should be the 'official' and administrative languages, respectively. Indonesian (Bahasa) is widely spoken and, while English is not discouraged (indeed, it is called a 'working' language), the develo pment of the nation, especially in education, will be restricted by lack of easy communication with external agencies and internet use.

At present the country has some 30 indigenous medical practitioners; half of them appear to be employed in an administrative capacity, running rural clinics. The country still depends on the provision of medical practitioners from countries such as the Philippines to support rural health clinics. In terms of developing a medical infrastructure and ongoing medical education, East Timor will require support from nations such as Portugal, Australia and Japan. East Timor will train nurses locally, initially with international support, and, despite the earlier problems with Indonesia, will need to train doctors at Indonesian universities because of their proximity, cost and cultural and linguistic compatibilities.

\section{Conclusions}

East Timor is emerging from a period of chaos, which saw the destruction of most of its infrastructure during the post-election turmoil in 1999. A mental health service has recently been established, with 16 nurse case managers employed largely in rural centres, within general medical clinics. These nurses have received 10 days of intensive formal training in psychiatry and ongoing intermittent supervision to equip them in a basic way to deal with the more serious, low-prevalence disorders using a handful of generic psychotropic medications. It is our experience that training a small cadre of specialist nurses was essential to initiating mental health services. The task remaining is to transmit the skills acquired by these specialist workers to a wider array of generic nurses in the community.

A recent formal review of this mental health service indicates it is functioning in an effective and cost-efficient fashion, treating serious mental disorders for the first time in a very impoverished nation. The health service faces many long-term problems in delivering healthcare to a very dispersed rural economy with extremely limited resources. The challenge is to institutionalise mental health as a priority service in East Timor and to ensure its sustainability with minimal external support.

\section{References}

Silove, D. (1999) Health and human rights of the East Timorese. Lancet, 353, 2067.

Zwi, A. \& Silove, D. (2002) Hearing the voices: mental health services in East Timor. Lancet, 360 (suppl.), s44-s46.

\title{
The European Society for Child and Adolescent Psychiatry (ESCAP): history and challenges for the future
}

\section{Ernest Caffo ${ }^{1}$ and Luisa Strik Lievers ${ }^{2}$}

\author{
${ }^{1}$ Professor of Child Neuropsychiatry, University of Modena and Reggio Emilia, Italy, and President of the \\ European Society for Child and Adolescent Psychiatry (ESCAP), email caffo@unimore.it \\ ${ }^{2}$ Child neuropsychiatrist, University of Modena and Reggio Emilia, Italy
}

\begin{abstract}
he European Society for Child and Adolescent Psychiatry (ESCAP) assembles 29 national societies of child and adolescent psychiatry of several countries belonging to the European Union or to its cultural and geographical area. It is the only association that gathers European psychiatrists who work with children and adolescents.

European child psychiatry is a dynamic entity. Its richness is progressively increasing, following the enlargement of the European U nion. ESCAP includes among its membership countries that have joined the U nion recently (such as Estonia, the Czech Republic and H ungary), some other countries that have not yet joined (such as Iceland and Turkey) and others that share
\end{abstract}

similar cultural roots (such as Israel). The Association also publishes a scientific journal, European Child and Adolescent Psychiatry.

\section{Origins and congress}

ESCAP has its roots in the UEP (Union of European Paedopsychiatrists), an association whose first meeting was held in Magglingen (Switzerland) in 1954 and that was officially established in 1960, during its first congress in Paris. Since then, congresses have been held regularly every 3-4 years and have focused on the more clinically relevant, complex and current topics. D uring each congress, a general assembly takes place to
For contributions to the

'Associations and collaborations' column, please contact John Henderson, email john.henderson53@ btopenworld.com

experience that training a small cadre of specialist nurses was essential to initiating mental health services. The task remaining is to transmit the skills acquired by these specialist workers to a wider array of generic nurses in the community.

\section{a}

ESCAP website: www.dgkjp.de/ escap/ 
elect the new board of the Society for the following 4 years. The board includes the President, Vice-President, Secretary and Treasurer. Many congresses have been organised. In Rome, in 1963, the congress topic was 'Character disorders in childhood and adolescence'; in Lausanne, in 1983, it was 'Aggression and the family'. More recently, in 1999, under the presidency of Professor H elmuth Remschmidt, the congress ' $\mathrm{N}$ ew challenges, new solutions' was held in Hamburg and the last one, in 2004, under the Presidency of Professor Philippe Jeammet, was organised in Paris on the subject of 'D evelopmental psychopathology - transmission and change'. The next ESCAP congress (the 13th) is proposed to be held in Florence in autumn 2007, under the new Presidency of Professor Ernesto Caffo.

\section{Aims}

According to its statutes, ESCAP has the following aims:

$O$ to foster the European tradition of child and adolescent psychiatry

0 to facilitate and extend the bonds between physicians practising child and adolescent psychiatry in European countries

0 to spread the results of research and experience in this branch of medicine by publishing reports and organising scientific conferences and meetings

o to collaborate with international organisations with

\section{the same or related goals. \\ Mental health problems are a public health issue that Europe has to face. \\ Roots of European child and adolescent psychiatry} Mental disorders are widespread in all European countries. Their incidence is rapidly increasing and the situation is projected to worsen in the next few years.
The culture of modern child psychiatry was born and developed in Europe and it later spread to other countries. Different approaches have been developed over many years, during which the European child psychiatric scenario has become rich and multifaceted (Remschmidt \& Van Engeland, 1999). Among the main approaches there are:

$O$ the tradition of child psychiatry based on a unified model of psychiatry and neurology, in countries such as Germany, France and Italy and in Eastern Europe (in Italy the discipline, founded by Giovanni Bollea, keeps the name of child neuropsychiatry)

o the remedial clinical tradition, which evolved into psychosomatic medicine, largely in Austria and Switzerland

O the psychodynamic/psychoanalytic approach, typical of Western Europe and of many schools, such as the French school of Serge Lebovici

o the empirical, epidemiological and statistical tradition (largely influenced by the United States), particularly in the U nited Kingdom (with the contribution of Professor Sir Michael Rutter), Scandinavia, Germany and Switzerland.

European child psychiatry offers wide psychotherapeutic (individual or group), pharmacological and psychosocial strategies. The richness of European child psychiatry arises from the dynamic coexistence of these different facets. By recognising the value of each of these different approaches, child psychiatry will be able to develop a versatile model of care. In fact, in European child psychiatry the focus is no longer on theoretical models but on the patient and the disorder.

What the different approaches to child and adolescent psychiatry in Europe have in common is the respect of children's rights. This is achieved in clinical practice by respecting the right that everybody has to receive adequate treatment, by helping families, by activating a social network to reinforce support and by informing the patient about the therapy and its goals through informed consent.

Typical of the European tradition is investment in the social and public field, by creating an active social network that involves several agencies able to offer support to everyone, of any age, gender and social and cultural background. In Europe, child psychiatrists work not only in a therapeutic direction, but also in terms of prevention, by respecting individual differences. In the third millennium Europe wants to be more open to different cultures. Migration from neighbouring as well as from more distant countries (mainly Africa and Asia) makes our system (and demands it to be) more flexible to respond to new cultures and habits.

\section{Child and adolescent mental health in Europe}

Mental health problems are a public health issue that Europe has to face. Mental disorders are widespread in all European countries. Their incidence is rapidly increasing and the situation is projected to worsen in the next few years. According to the World Health O rganization (2005), in Europe $80 \%$ of young people report psychological well-being. However, that means that one adolescent in five has cognitive, emotional and behavioural difficulties and one adolescent in eight suffers from a diagnosable mental disorder. Among the most prevalent and discussed disorders are attentiondeficit hyperactivity disorder (ADHD), anxiety and depression (a third of depressed adults are estimated to have shown the first episode before 21 years of age), eating disorders, pervasive develo pmental disorder and learning disability. Developmental psychiatric disorders are unlikely to have a spontaneous remission, cause problems with social adaptation and are associated with mental disorder in adult life if they are not diagnosed and treated early.

\section{Child and adolescent psychiatry in Europe today}

The branch of psychiatry devoted to children and adolescents is recognised at present as a specialisation or sub-specialisation in all European countries. Since 1994, child psychiatry inside the Union of European Medical Specialists (UEMS) has been a separate discipline from psychiatry and has its own section and board - Child and Adolescent Psychiatry/Psychotherapy 
(CAPP) (Hill \& Rothenberger, 2004). It is strongly linked to other disciplines, such as paediatrics, neurology, psychiatry and psychology, and to many others involved in children's physical and mental health, such as pedagogy, rehabilitation, logopaedics and physiotherapy. This interdisciplinary work is fundamental for prevention, treatment and research.

The member states of the European U nion differ in their organisation of child and adolescent mental health services and in child psychiatry training. There are those that prefer an affiliation with adult psychiatry (e.g. France) and others that choose training immediately focused on developmental age, but including a neurological approach (e.g. Italy). For students, ESCAP foresees at least 12 months of practice in an adult psychiatric department, an optional experience in neurology departments and theoretical and practical knowledge in the paediatric field (H ill \& Rothenberger, 2004).

An important challenge for a child psychiatrist is involvement in research, into both the pathogenesis of disorders and their treatment. Evidence-based medical practice is another challenge. In the last 20 years, the three main areas of child psychiatry research have been:

o physiology (molecular biology, genetics, neuroimaging, pharmacology)

o child development (developmental psychology, psychopathology, neurology)

o assessment measures.

\section{Perspective for the future}

W hile balancing different national and ethnic realities, Europe has to face and overcome important challenges for the development of psychiatry, and more specifically of child and adolescent psychiatry. The main ones are developing effective and timely interventions, improving the accessibility and quality of services, and investing in primary and secondary prevention. In addition, those interventions, both therapeutic and preventive, individual and community, increasingly have to be based on scientific evidence. At the same time, a system of efficacy and efficiency checks on results and procedures has to be established; at present this is still largely lacking. To achieve this goal, it is useful to compare the experiences of different countries, during open discussions at congresses and through scientific journals. The main aim is to create European guidelines and protocols, but as a set of flexible and dynamic instruments. Such a protocol could be evaluated in each country. The N orth American model should no longer be simply imported and applied to the different European cultural settings. A tentative effort in this direction was made during the conference 'C aring for children in times of war, terrorism and disaster' organised by ESC AP, Foundation Child and the International Association for Child and Adolescent Psychiatry and Allied Professions (IACAPAP) in Rome in July 2003. At the end of the meeting, which involved experts in the field of childhood trauma, the Declaration of Rome was endorsed, in order to summarise the key points of the conference (see Box 1).

\section{Box 1. Declaration of Rome}

At the meeting of ESCAP, Foundation Child and the IACAPAP in Rome in July 2003 , on childhood trauma, the following principles were articulated:

- Care for children affected by war, terrorism, disaster and maltreatment should respect the culture of the child and society, respect individual differences, promote their reintegration into family and society, and enhance their normal development.

- The biological changes to the brain associated with trauma must be appreciated as a significant influence on physical brain development and behaviour. As knowledge advances in these areas, treatments will need to incorporate the means to ameliorate these biological changes.

- The trauma of maltreatment, war, terrorism and disaster is intergenerational. Care needs to be provided not only at the time of the trauma but also in its aftermath, if later generations are not to be affected.

- Governments should increase their investment in prevention, treatment and research in the area of caring for children affected by maltreatment, war, terrorism and disaster. Government officials and agencies responsible for health, education and social services need to collaborate to anticipate and prepare for the integrated responses needed in the aftermath of traumatic events. Government leadership is needed to facilitate inter-group understanding and reconciliation.

- The UN Convention on the Rights of the Child must be seen as applicable to children subject to maltreatment and affected by war, terrorism and disaster. Governmental bodies, at all levels, need to recognise and incorporate the protections and entitlements afforded by the UN Convention on the Rights of the Child. Policies and programmes should embrace the rights articulated in that Convention.

- Policy makers and carers should be informed of the latest findings from research, to inform the development of systems of care that afford responsive and responsible care for children.

- Ideally, the trauma of war and terrorism can be diminished and ultimately prevented through education, negotiation, mutual respect for rights and enhanced personal understanding. In the process of providing care, every effort should be made to foster a climate that will lessen the likelihood of continuing and future conflict. An accurate depiction of the dimension of human suffering following these events should be aired in the media.

- Legislators, clinicians and the general public should be educated about the public health problems associated with emotional, physical and sexual abuse, and about the recognition and treatment of children and families affected by child maltreatment.

- Care for children is an investment in the long-term health and productivity of nations. Therefore, care, treatment and prevention should be facilitated by more international cooperation, involvement of governments, non-governmental organisations and international organisations.

The need to share the same goals for the promotion of mental health among all European Union countries, despite their differing socio-political organisation and resources, is strongly supported by the World $\mathrm{H}$ ealth $\mathrm{O}$ rganization (WHO). To this end, to gether with the European U nion and the Council of Europe, the WHO European Regional 0 ffice organised a ministerial
The member

states of the

European Union differ in their organisation of child and

adolescent mental

health services

and in child

psychiatry

training. There are

those that prefer

an affiliation with

adult psychiatry

(e.g. France) and

others that choose

training

immediately

focused on

developmental

age, but including

a neurological

approach (e.g.

Italy).

The main aim is to create European guidelines and protocols, but as a set of flexible and dynamic

instruments. Such

a protocol could

be evaluated in

each country. The

North American model should no

longer be simply

imported and

applied to the different

European cultural settings. 
conference in Helsinki, 12-15 January 2005, which was open to 52 European member states and professional and non-governmental organisations. The preparatory work for this meeting began in 2003 in Copenhagen and was developed in the course of regular meetings (Athens, Brussels, Paris, Moscow and, more recently, Luxemburg and Tallin). The final documents of the conference contain an analysis of the current state of European psychiatry and some suggestions on how to improve treatment efficacy and efficiency, how to increase service quality and accessibility, and how to overcome stigma and protect human rights (World Health O rganization, 2005).

\section{Future challenges: research and training}

A fundamental challenge in the future of child psychiatry is further research, particularly into:

0 the aetiology and pathogenesis of psychopathological disorders in children and adolescents

O symptoms and disorders, stability and change

o externalising and internalising disorders

o developmental disorders

o evidence-based treatments and interventions, as well as preventive strategies (involving also the family and the more extended environment).

Another challenge concerns the training of young child psychiatrists and researchers. ESCAP, in collaboration with Foundation Child and the International Association for Child and Adolescent Psychiatry and the IACAPAP, has organised a research seminar in Italy every year over the last 5 years, which gathers leading professionals in child and adult psychiatry from all over the world. The research seminar is open to 35-40 young European child psychiatry students, sponsored by
Foundation Child, to spend a week of study in order to be trained in a particular area, which changes every year (mood and anxiety disorders, treatment evaluation, etc.). In addition, every student is invited to present to the group his/her past or current research projects in order to get useful feedback from colleagues and senior supervisors. The next one is to be held from 10 to 15 April 2005 in Bocca di Magra (La Spezia).

For such scientific activities, for facilitating a continuous exchange of scientific knowledge and developing a common system of training, European child psychiatry needs to be supported by adequate funds and structures.

Scientific communication, both intra- and inter-disciplinary, should also be facilitated through the creation of international research projects, scientific meetings and scientific publications. Since knowledge in the field of child and adolescent mental health is constantly evolving, regular professional updating is necessary.

\section{References and sources}

Bollea, G. (1979) Psichiatria dell'eta evolutiva: fattori etiopatogenetici. Rome: Bulzoni.

Hill, P. \& Rothenberger, A. (2004) Can we - and should we have a 'Euro-psychiatry' for children and adolescents? The work of the UEMS Section and Board for Child and Adolescent Psychiatry/Psychotherapy. International Psychiatry, no. 6, 20-21.

Lebovici, S., et al (1999) Nouveau traité de psychiatrie de l'enfant et de l'adolescent. Paris: Presses U niversitaires de France.

Remschmidt, H. \& Van Engeland, H. (eds) (1999) Child and Adolescent Psychiatry in Europe: Historical Development, Current Situation, Future Perspectives. D armstadt: Steinkopff. Rutter, M. \& Taylor, E. (eds) (2002) Child and Adolescent Psychiatry, Vol. IV. O xford: Blackwell.

World Health Organization (2005) Final documents of the ministerial conference, Helsinki, 12-15 January 2005: Mental Health Action Plan for Europe and European Declaration on Mental Health. Available at www.euro.who.int/ mentalhealth2005.

\section{The College \\ website}

(www.rcpsych.ac.uk)

has seen useful

changes, so that

links with the

Board of Inter-

national Affairs,

International

Psychiatry and

international

mental health

organisations are

much more

accessible.

Suggestions for

further improve-

ments are

welcome. Contact

jcarroll@rcpsych.ac.uk

NEWS, NOTES, FORTHCOM ING INTERNATIONAL EVENTS

\section{News and notes}

For contributions to this column, please contact Brian Martindale FRCPsych, Early Intervention in Psychosis Service, Monkwearmouth Hospital, Newcastle Road, Sunderland SR5 1NB, email drbmartindale@blueyonder.co.uk

\section{International programme for the College Annual M eeting in Edinburgh}

The Board of International Affairs has organised a stimulating programme within the College Annual Meeting in Edinburgh this year. The highlight will be the visit of the President of the World Psychiatric Association (WPA), Professor 0 kasha, who will be involved in a major discussion on Wednesday 22 June on 'The recruitment and migration of psychiatrists from developing countries: an ethical issue and a conflict between social responsibility and individual freedom of choice'. Some of the new international divisions are organising a day-long programme for Thursday 23 June. The themes will centre on the diversity of issues in psychiatry in different cultures and settings, and implications for training.

\section{The European Task Force and progress in the evaluation of CME in Europe}

The European Task Force - comprising official representatives of the Association of European Psychiatrists, the Section and Board of the Psychiatry subsection of the 